A SOS é mais frequente em indivíduos do sexo masculino e geralmente é unilateral, ocorrendo bilate-

\title{
ऽ Síndrome de Osgood-Schlatter
}

Dr. Cláudio Espírito Santo ${ }^{1}$, Dra. Joana Rodrigues $\mathrm{Maia}^{2}$, Dr. Diogo Lino Moura ${ }^{3}$, Prof. Doutor Fernando

\section{Fonseca}

${ }^{1}$ Assistente de Medicina Geral e Familiar, USF Rainha Santa Isabel; ${ }^{2}$ Médica interna de Medicina Geral e Familiar, UCSP Cantanhede; ${ }^{3}$ Médico interno de Ortopedia do Serviço de Ortopedia do Centro Hospitalar e Universitário de Coimbra (CHUC); Faculdade de Medicina da Universidade de Coimbra (FMUC); ${ }^{4}$ Diretor do Serviço de Ortopedia do CHUC, FMUC. Coimbra

\section{RESUMO / ABSTRACT}

A Síndrome de Osgood-Schlatter (SOS) carateriza-se pela separação parcial entre a epífise tibial e o centro de ossificação da tuberosidade anterior da tíbia, constituindo a causa mais comum de morbilidade do joelho durante a adolescência. Apresenta particular frequência nos indivíduos do sexo masculino praticantes de exercício físico, com idades compreendidas entre os 12 e os 15 anos. Esta patologia é autolimitada, de resolução espontânea em 90\% dos pacientes, no entanto pode dar sintomas na idade adulta e ser até necessário tratamento cirúrgico. Neste artigo apresentamos uma revisão da literatura científica acerca da definição, clínica, diagnóstico e tratamento da SOS.

The Osgood-Schlatter syndrome (OSS) is characterized by partial separation between tibial epiphysis and the anterior tibial tuberosity ossification center, establishing itself as the most common origin of morbidity localized to the knee during adolescence. It is characterized by its particular frequency in males aged between 12 and 15 years and physical exercise practitioners. This condition is self-limited, with an expected spontaneous recovery in $90 \%$ of patients. However, symptoms can happen at adult stage and surgical intervention can be needed. In this paper we review the scientific literature about definition, clinic, diagnosis and treatment of SOS.

\section{PALAVRAS-CHAVE / KEYWORDS}

Síndrome de Osgood-Schlatter, dor joelho, tuberosidade tibial Osgood-Schlatter syndrome, knee pain, tibial tuberosity

\section{Definição}

A Síndrome de Osgood-Schlatter (SOS), também conhecida por osteocondrose ou apofisite de tração da tuberosidade anterior da tíbia, caracteriza-se pela avulsão parcial da apófise da tuberosidade anterior da tíbia provocada pela tração contínua exercida pelo aparelho extensor do joelho. Foi pela primeira vez descrita em 1903, de forma independente e simultânea por dois cirurgiões ortopédicos, o norte-americano Robert Osgood e o suíço Carl Schlatter. ${ }^{1-3}$

\section{Epidemiologia}

A SOS é a causa mais comum de morbilidade localizada no joelho na adolescência. ${ }^{1}$ A maioria dos casos de SOS ocorre em adolescentes praticantes de exercício físico, por solicitarem mais o aparelho extensor da perna e, consequentemente, o seu ponto de inserção distal: a tuberosidade anterior da tíbia ainda imatura. Os estudos de osteocondrose em atletas indicam que aproximadamente $20 \%$ dos adolescentes praticantes de desporto apresentavam sintomas desta condição, em comparação com a incidência de $4.5 \%$ em não praticantes. ${ }^{4}$

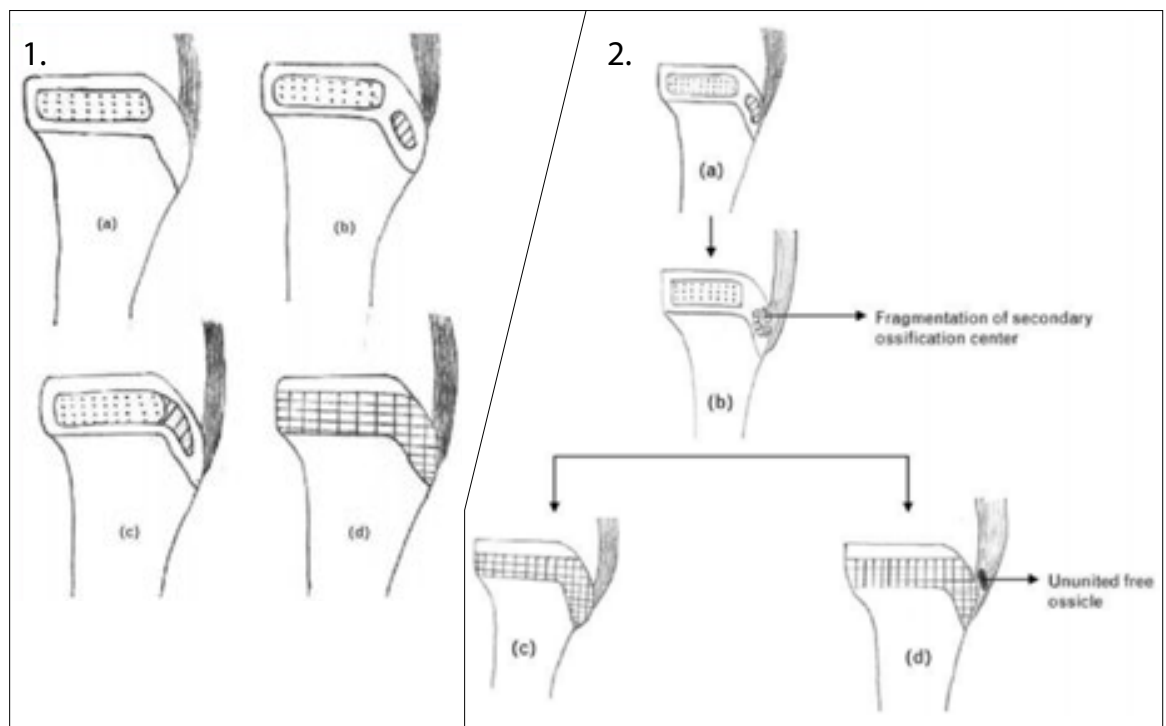

Figura 1 - Estadios radiológicos de Ehrenborg e Lagergren da maturação da tuberosidade tibial. a) Estadio Cartilaginoso (0-11 anos de idade); b) Estadio Apofisário (11-14 anos de idade); c) Estádio Epifisário, coalescência da apófise e epífise tibiais (14-18 anos de idade); d) Estádio Ósseo, epífise fundida (>18 anos de idade)..$^{8-12}$ ralmente em cerca de 20 a 30\% dos casos. No sexo masculino, a idade habitual de apresentação situa-se entre os 12 e os 15 anos, enquanto no sexo feminino se situa entre os 8 e os 12 anos. $^{5-7}$

\section{Fisiopatologia}

O centro de ossificação secundário da tuberosidade anterior da tíbia aparece entre os 11 e 14 anos de vida e funde-se à epífise tibial superior entre os 14 e os 18 anos. Antes de ocorrer esta fusão, os dois centros de ossificação estão separados apenas por cartilagem, sendo que é neste período de fragilidade local que ocorre a SOS (estadio apofisário dos Estadios Radiológicos de Ehrenborg e Lagergren da maturação da tuberosidade tibial - Figura 1). ${ }^{8-12} \mathrm{~A}$ forte contração muscular e a tração contínua efetuadas pelo aparelho extensor do joelho, particularmente evidentes durante a prática de exercício físico intenso, são superiores à resistência do seu ponto de inserção distal, o frágil centro de ossificação da tuberosidade anterior da tíbia no adolescente, que ainda está separado da epífise tibial superior apenas

2.

Figura 2 - Patogenia da Síndrome de Osgood-Schlatter: a) aparência do centro de ossificação secundário da tuberosidade tibial; b) fragmentação do centro de ossificação; c) fusão e ossificação do centro de ossificação da tuberosidade tibial à epífise tibial superior, resultando em tuberosidade proeminente; d) ossículo livre não unido. ${ }^{8}$ 
por cartilagem não ossificada. Esta desproporção entre fortes tendões e apófises de inserção imaturas, típica da idade pediátrica, conduz a inflamação e avulsão parcial do centro de ossificação secundário da tuberosidade anterior da tíbia. A avulsão pode ocorrer tanto durante a fase de pré-ossificação, como após ossificação do centro de ossificação secundário, ocorrendo precisamente na região cartilagínea frágil que separa o centro de ossificação da tuberosidade anterior da tíbia da epífise tibial superior. Esta separação do osso ou da cartilagem não impede o crescimento e ossificação, o que irá provocar uma ossificação saliente da tuberosidade anterior da tíbia ou a presença de um ossículo anterior isolado que corresponde ao seu centro de ossificação parcialmente avulsionado. ${ }^{9-11}$

\section{Etiologia}

A etiologia da SOS é desconhecida, embora existam diversos estudos que associam esta patologia a algumas variações que aumentam a força de tração do aparelho extensor sobre a tuberosidade anterior da tíbia: pés em pronação, joelhos valgos e em rotação medial e patela alta. ${ }^{13-15}$

\section{Apresentação clínica}

A sintomatologia pode variar desde um ligeiro desconforto local a dor incapacitante. Habitualmente caracteriza-se por dor vaga, com agravamento gradual, e tumefação localizadas à região da tuberosidade tibial. Inicialmente, a dor é moderada e intermitente. Em fase aguda, a dor é severa, contínua e acompanhada de incapacidade funcional. A dor agrava após a prática de desporto, principalmente se este envolver a realização de salto e corrida (por exemplo, basquetebol, voleibol, corrida) e/ou contacto direto, como por exemplo ajoelhar. ${ }^{16} \mathrm{~A}$ dor é mais frequentemente mecânica, isto é, alivia com paragem da solicitação do aparelho extensor.

Assim, o quadro típico envolve um adolescente do sexo masculino, com idade compreendida entre os 12 e os 15 anos, praticante de exercício físico, que apresenta queixas de dor e tumefação localizadas na região da tuberosidade tibial, com início gradual e agravamento após a realização de atividade física. Durante a fase aguda, a observação clínica revela sensibilidade, tumefação e proeminência da área da tuberosidade tibial. A dor é reprodutível realizando palpação local e extensão do joelho contra resistência, teste que solicita o aparelho extensor e tração sobre a tuberosidade anterior da tíbia. Se o paciente for incapaz de fazer extensão ativa da perna deve-se ponderar avulsão completa da tuberosidade anterior da tíbia ou comprometimento do aparelho extensor tendinoso. Após a fase aguda, a dor e a tumefação geralmente desaparecem, persistindo como único sinal a presença de uma tuberosidade anterior da tíbia proeminente (Figura 3). ${ }^{5-8,16,17}$

\section{História natural}

A grande maioria dos investigadores refere que a SOS se carateriza como uma patologia autolimitada, de resolução espontânea, com recuperação esperada em cerca de $90 \%$ dos pacientes. Aproximadamente 10\% dos pacientes irá manter os sintomas até a idade adulta, apesar de tratamento conservador. ${ }^{5-7,16,17}$

\section{Diagnóstico diferencial}

Em todos os pacientes jovens, praticantes de exercício físico que apresentem gonalgia, de início gradual, com agravamento durante e após a prática de exercício físico, seja uni ou bilateral, deve ponderar-se, além da SOS, os seguintes diagnósticos: Sindrome Sinding-Lansen-Johansson, osteocondrose semelhante, mas que atinge o pólo distal da patela,

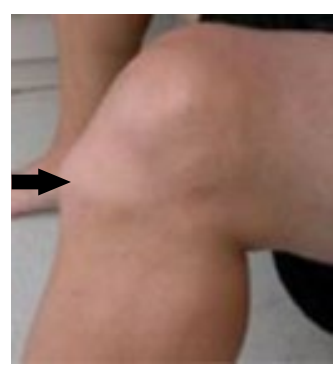

Figura 3 A observação lateral do joelho demonstra a tuberosidade anterior da tíbia proeminente numa situação de SOS (seta)
Síndrome de Hoffa, inflamação da bolsa adiposa infrapatelar, e fratura da tubersidade anterior da tíbia. A dor patelofemoral, tumores ou infeções são também diagnósticos a colocar. ${ }^{18-19}$

\section{Exames complementares de diagnóstico}

O diagnóstico da SOS é essencialmente clínico, sendo raramente necessário o recurso a exames complementares. ${ }^{8}$ A radiografia do joelho em incidência perfil, com o membro inferior em $10-20^{\circ}$ de rotação medial, pode demonstrar a presença de irregularidade da tuberosidade anterior da tíbia, com presença de avulsão nos estádios iniciais ou mesmo fragmentação nos processos mais avançados. Em alguns casos, mesmo após a fusão e encerramento do centro de ossificação secundário, pode ser detectada a existência de um ossículo anterior único isolado (Figura 4). A ecografia da articulação do tendão patelar e da sua inserção tibial pode também ser um método de complementar o diagnóstico. A realização de ressonância magnética poderá estar indicada quando, após a avaliação clínica e imagiológica, permaneçam dúvidas diagnósticas. ${ }^{4,6-8,12,15,19}$

\section{Tratamento}

Não existem quaisquer estudos prospetivos, controlados ou aleatorizados que avaliem diretamente qual o melhor tratamento para a SOS. Assim sendo, na literatura apenas se encontram estudos retrospetivos sobre qual a atitude a tomar perante o diagnóstico desta patologia. O tratamento sintomático é o recomendado numa fase precoce. Na fase aguda álgica deve ser feita limitação da atividade física e

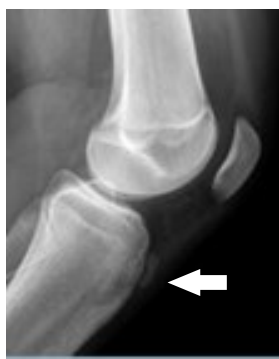
Figura 4 - Radiografia do joelho em incidência perfil demonstrando ossículo ao nível da tuberosidade tibial, alterações típicas da SOS (seta) 
prescritos métodos anti-inflamatórios, tais como gelo local, terapêutica anti-inflamatória oral e joelheiras de proteção. A atividade física não deve ser interrompida na totalidade caso o paciente a consiga suportar. Assim, pacientes com dor moderada e sem impotência funcional deverão manter a prática de exercício regular, associando a aplicação de uma joelheira de proteção e um anti-inflamatório não-esteroide. Nos pacientes com dor severa, associada ou não a impotência funcional, deverá ser limitada por uns dias a prática de exercício físico. ${ }^{6,8,13} \mathrm{~A}$ fisioterapia e um programa de condicionamento e fortalecimento muscular correspondem a uma parte importante da abordagem terapêutica da SOS. Estas deverão ter início logo após o fim da fase aguda da doença, estando recomendados exercícios de fortalecimento e de aumento da flexibilidade da musculatura regional envolvida na mobilidade da articulação do joelho. Inicialmente estão indicados exercícios isométricos de baixa intensidade, sendo gradualmente instituídos movimentos de intensidade crescente. Alguns estudos demonstram que cerca de 90\% dos pacientes responde bem à combinação da aplicação de gelo, anti-inflamatório oral e repouso relativo. ${ }^{20-22}$ No entanto, em cerca de 5 a 10\% dos casos, os sintomas mantêm-se e poderá estar indicado tratamento cirúrgico. ${ }^{16,20}$

Existem diversas técnicas cirúrgicas descritas para o tratamento desta patologia, com resultados diversos, não existindo um procedimento que seja comprovadamente mais eficaz que os restantes (Figura 5).5,16,20,23-25 Mital et. al apresentam resultados de intervenções cirúrgicas a 15 joelhos com SOS.

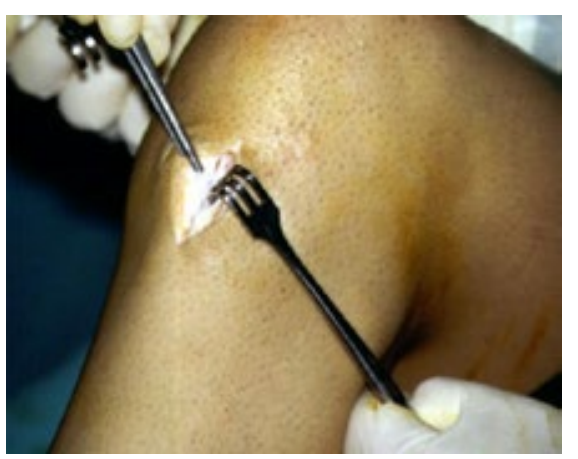

Figura 5 - Exérese de ossículo da tuberosidade anterior da tíbia em SOS.
Todos apresentavam ossículo livre separado, dor e sensibilidade local, mantendo-se sintomáticos por um período médio de 3.8 anos, apesar de instituídas medidas terapêuticas conservadoras. Segundo os autores, todos os pacientes referiram melhoria da dor após o procedimento cirúrgico excisão do ossículo e da bolsa sinovial adjacente. ${ }^{20}$

Flowers e Bhadreswar utilizaram uma modificação da técnica clássica de Ferciot e Thomson de excisão da proeminência da tuberosidade da tíbia, acrescentando-lhe a remoção de qualquer material osteocartilagíneo presente. Referem alívio da dor em 95\% dos pacientes e melhoria da proeminência em $86 \%$ dos pacientes. ${ }^{16,23,24}$

\section{Complicações}

Está descrito que em cerca de 10\% dos casos de SOS podem ocorrer complicações. A pseudartrose e a migração e persistência de um ossículo livre dentro do tendão patelar podem originar um encerramento prematuro da porção anterior da epífise tibial superior, resultando a deformidade em recurvatum do joelho. Como complicações podem também ocorrer patela alta e alterações na biomecânica da articulação patelofemoral, situações que podem conduzir a instabilidade patelar e gonartrose precoce. Estão ainda descritas situações de fragilidade da inserção do tendão patelar na tuberosidade anterior da tíbia que culminam em avulsões do mesmo. 8,26

\section{Conclusão}

A SOS é na maior parte das vezes uma patologia autolimitada, mas cujo diagnóstico é necessário reconhecer, na medida em que afeta a capacidade física e desportiva. A sua fisiopatologia explica a maior ocorrência na adolescência e a sua clínica típica permite diagnóstico fundamentado sobretudo pela sintomatologia. A radiografia pode permitir confirmar o diagnóstico e excluir diagnósticos diferenciais. O início de tratamento anti-inflamatório e da limitação parcial da atividade física são fundamentais para permitir a recuperação rápida do adolescente à atividade física habitual. Estas medidas serão eficazes na grande maioria dos casos, sendo que em apenas 5 a 10\% destes a sintomatologia irá subsistir até à idade adulta, podendo estar indicado tratamento cirúrgico.

Os autores declaram ausência de conflito de interesses.

\section{Correspondência:}

Dr. Cláudio Espírito Santo

claudioespsanto@gmail.com

USF Rainha Santa Isabel, Coimbra

\section{Bibliografia}

1. Osgood RB. Lesions of the tibial tubercle occurring during adoloscence. Boston Med Surg J 1903; 148:114-117.

2. Schlatter C. Verletzungen des schnabelformigen Fortsatzes der oberen Tibiaepiphyse. Beitr Klin Chir 1903; 38:874-887.

3. Kujala, U. M., Kvist, M., and Osterman, K., Knee injuries in athletes. Review of exertion injuries and retrospective study of outpatient sports clinic material. Sports Med. 1986; 3(6):447-60.

4. Kujala, U. M., Kvist, M., and Heinonen, O., Osgood-Schlatter's disease in adolescent athletes. Retrospective study of incidence and duration. Am J Sports Med. 1985; 13(4):236-41.

5. Orava, S., Malinen, L., Karpakka, J., Kvist, M., Leppilahti, J., Rantanen, J., and Kujala, U. M., Results of surgical treatment of unresolved Osgood-Schlatter lesion. Ann Chir Gynaecol. 2000; 89(4):298-302.

6. Bloom, O. J., Mackler, L., and Barbee, J., Clinical inquiries. What is the best treatment for Osgood-Schlatter disease? J Fam Pract. 2004; 53(2):153-6.

7. Blankstein, A., Cohen, I., Heim, M., Diamant, L., Salai, M., Chechick, A., and Ganel, A., Ultrasonography as a diagnostic modality in Osgood-Schlatter disease. A clinical study and review of the literature. Arch Orthop Trauma Surg. 2001; 121(9):536-9.

8. Gholve, P. A., Scher, D. M., Khakharia, S., Widmann, R. F., and Green, D. W., Osgood Schlatter syndrome. Curr Opin Pediatr. 2007; 19(1):44-50.

9. Ehrenborg, G., The Osgood-Schlatter lesion. A clinical study of 170 cases. Acta Chir Scand. 1962; 124:89-105.

10. Ehrenborg, G., and Engfeldt, B., Histologic changes in the Osgood-Schlatter lesion. Acta Chir Scand. 1961; 121:328-37.

11. Ehrenborg, G., and Engfeldt, B., The insertion of the ligamentum patellae on the tibial tuberosity. Some views in connection with the Osgood-Schlatter lesion. Acta Chir Scand. 1961; 121:491-9.

12. Ehrenborg, G., and Lagergren, C., Roentgenologic changes in the Osgood-Schlatter lesion. Acta Chir Scand. 1961; 121:315-27.

13. Willner, P., Osgood-Schlatter's disease: etiology and treatment. Clin Orthop Relat Res. 1969; 62:178-9.

14. Gigante, A., Bevilacqua, C., Bonetti, M. G., and Greco, F., Increased external tibial torsion in Osgood-Schlatter disease. Acta Orthop Scand. 2003; 74(4):431-6. 
15. Aparicio, G., Abril, J. C., Calvo, E., and Alvarez, L., Radiologic study of patellar height in Osgood-Schlatter disease. J Pediatr Orthop. 1997; 17(1):63-6.

16. Flowers, M. J., and Bhadreshwar, D. R., Tibial tuberosity excision for symptomatic Osgood-Schlatter disease. J Pediatr Orthop. 1995; 15(3):292-7.

17. Ross, M. D., and Villard, D., Disability levels of college-aged men with a history of Osgood-Schlatter disease. J Strength Cond Res. 2003; 17(4):659-63.

18. Medlar, R. C., and Lyne, E. D., Sinding-Larsen-Johansson disease. Its etiology and natural history. J Bone Joint Surg Am. 1978; 60(8):1113-6.

19. Jacobson, J. A., Lenchik, L., Ruhoy, M. K., Schweitzer, M. E., and Resnick, D., MR imaging of the infrapatellar fat pad of Hoffa. Radiographics. 1997; 17(3):675-91.

20. Mital MA, Matza RA, Cohen J. The so-called unresolved Osgood-Schlatter lesion: a concept based on fifteen surgically treated lesions. J Bone Joint Surg Am 1980; 62:732-739.

21. Beovich R, Fricker PA. Osgood-Schlatter's disease a review of the literature and an Australian series. Aust J Sci Med Sport 1988; 20: 11-13.

22. Hussain A,f Hagroo GA. Osgood-Schlatter disease. Sports Exer Injury 1996; 2:202-206.

23. Ferciot CF. Surgical management of anterior tibial epiphysis. Clin Orthop 1955; 5:204-206.

24. Thompson JEM. Operative treatment of osteochondritis of the tibial tubercle. J Bone Joint Surg Am 1956; 38:142-148.

25. Glynn MK, Regan BF. Surgical treatment of Osgood-Schlatter's disease. J Pediatr Orthop 1983; 3:216-219

26. Vreju F, Ciurea P, Rosu A. Osgood-Schlatter disease-ultrasonographic diagnostic. Med Ultrason. 2010; 12(4):336-9. 